\title{
Astromineralogy: Crystalline silicates
}

\author{
F.J. Molster \\ ESTEC/ESA, RSSD-ST, Keplerlaan 1, 2201 AZ Noordwijk, The \\ Netherlands
}

\begin{abstract}
Silicates are the most abundant dust component in our Universe. For a long time it was generally assumed that around evolved stars only amorphous silicates were present. The infrared space observatory (ISO; Kessler et al. 1996) discovered that the abundance of crystalline silicates can be quite significant. Thanks to ISO it is now for the first time possible to determine the exact chemical composition of the silicates, and in contrast to the amorphous silicates, the crystalline silicates turned out to be very Fe-poor (i.e. forsterite and enstatite). Careful investigations of the spectra of crystalline silicates in several conditions, both in laboratory and in space, have taken place. It was found that not only the abundance of the crystalline silicates is related to the spatial distribution of the circumstellar dust, but also the shape of the features differs between sources with and without a disk-like dust distribution. Because of the many spectral features of the crystalline silicates one can easily determine temperatures and mass ratios for enstatite and forsterite. But even single features contain interesting information. The position and FWHM of the 69.0 micron forsterite feature, might be used as a new diagnostic of characteristic temperatures of (crystalline) silicate dust, while the 33.6 micron forsterite feature bears evidence for its formation history.
\end{abstract}

\section{Introduction}

Dust plays a very important role in our Universe. It is involved in many processes, like $\mathrm{H}_{2}$ formation in molecular clouds, the energy balance of the interstellar medium, star formation, and the efficiency of the stellar winds at the end of the life of stars. A good knowledge of the properties of dust will lead to a better understanding of these important processes. Dust is best studied in the infrared, because of the many ro-vibrational bands present in that wavelength range. Unfortunately for a long time the resolution and/or wavelength coverage of infrared camera's was not sufficient for detailed studies of astronomical dust. The launch of ISO changed this situation dramatically. For the first time it was possible to take spectra of stars and their circumstellar environment ranging from 2.4 to $200 \mu \mathrm{m}$. This unprecedented wavelength coverage together with the spectral resolution were ideal to study astronomical dust.

Thanks to the ISO spectra many minerals were discovered in evolved stars: diopside (Koike et al. 2000a), carbonates (Kemper et al. 2002a), FeS (Hony et al. this volume) crystalline $\mathrm{H}_{2} \mathrm{O}$ ice and silica (Molster, Waters \& Tielens 2002a). 


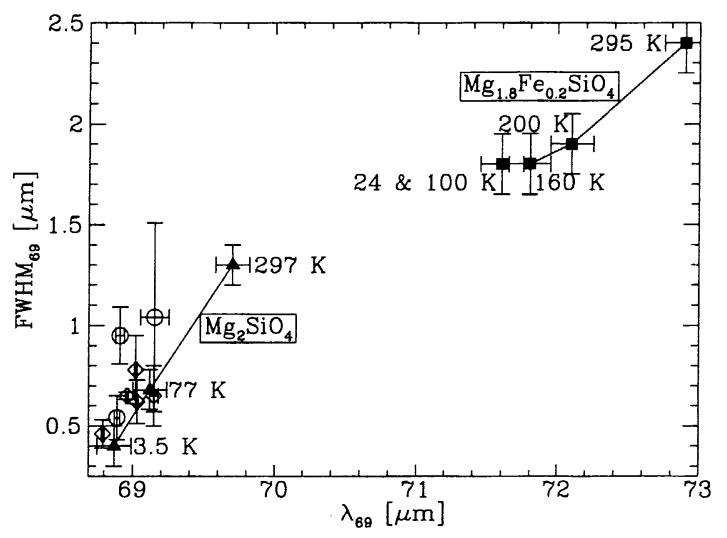

Figure 1. The observed FWHM and peak wavelength of the 69.0 micron forsterite feature in the spectra of the dust around evolved stars (open diamonds for the sources with a disk and open circles for the sources without a disk) and in the laboratory at different temperatures (filled triangles - forsterite $\left(\mathrm{Fo}_{100}\right.$; Bowey et al., 2000), and filled squares - olivine (Fo90; Mennella et al. 1998)). The temperatures are indicated at each point, and within the resolution the $24 \mathrm{~K}$ and $100 \mathrm{~K}$ for Fogo are similar. Figure taken from Molster et al. (2002b).

But, one of the greatest discoveries of ISO was the detection of crystalline silicates around evolved stars (Waters et al. 1996). The spectral resolution of ISO even allowed to get the exact chemical composition of these crystalline silicates (see Fig. 1). The majority of the crystalline silicates was formed by the Mg-rich endmembers of olivine (forsterite; $\mathrm{Mg}_{2} \mathrm{SiO}_{4}$ ) and pyroxene (enstatite; $\mathrm{MgSiO}_{3}$ ). Although, in general the crystalline silicates only contribute $5-10 \%$ of the mass, in some cases it is $\mathrm{p}$ to $75 \%$ (Molster et al. 2001).

The era of astromineralogy has been opened by the launch of ISO. In this contribution I will give an overview of the research of (crystalline) silicate dust around evolved stars, and present the most important results.

\section{Mineralogy of the crystalline silicates}

Although crystalline silicates do have intrinsically strong bands around $10 \mu \mathrm{m}$, ISO was necessary to detect them. They are simply to cold, at least colder than the amorphous silicates (see also Section 3.2). In Fig. 1, one can see that the crystalline olivine grains do not contain a significant amount of $\mathrm{Fe}$ (similarly a feature at $40.5 \mu \mathrm{m}$ can be used to proof the Fe-poorness of the pyroxene crystals). This reduces the absorptivity dramtically (see e.g. Dorschner et al. 1995). Therefore the temperature of the crystalline silicates will be very low and no significant feature wil be detected in the $10 \mu \mathrm{m}$ region (Fig. 2). At the same time amorphous silicates have a higher opacity due to small Fe-inclusions (Kemper et al. 2002b). This explains why amorphous silicates have a higher temperature than the crystalline silicates. This also results in the overwhelming 


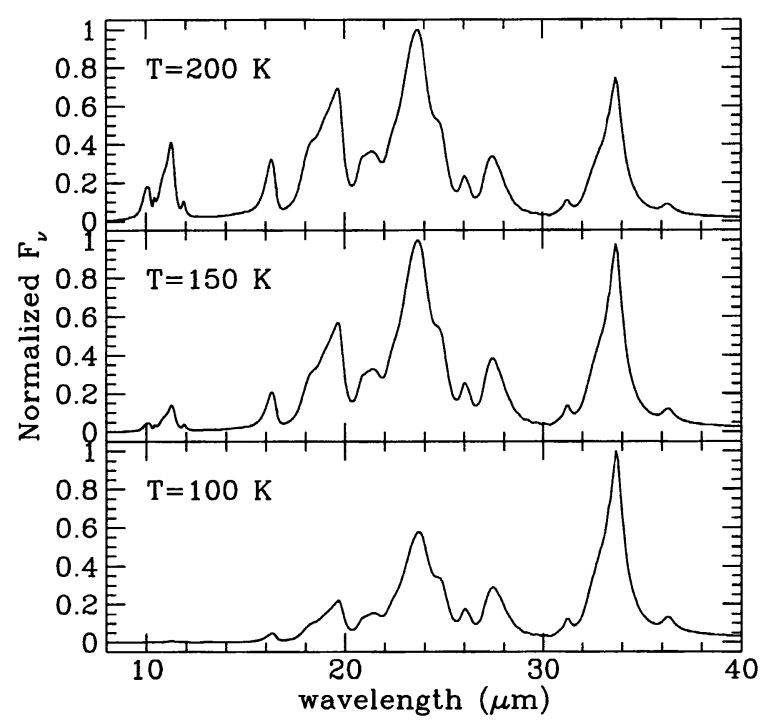

Figure 2. The laboratory transmission spectrum of forsterite, multiplied with a blackbody curve of 200,150 and $100 \mathrm{~K}$. Note the decrease in the strength of the peaks in the $10 \mu \mathrm{m}$ region when lowering the temperature

of the emission features of the crystalline silicates by the broad emission feature of the amorphous silicates in the $10 \mu \mathrm{m}$ region for low mass loss rates (i.e. optically thin $10 \mu \mathrm{m}$ region) as can be seen in Fig. $2 \& 3$ from Kemper et al. (2001). Both effects explain, why the crystalline silicates in (cold) circumstellar dust shells show only significant features beyond $20 \mu \mathrm{m}$, and why an instrument like ISO was necessary to detect them.

\section{Correlation study}

\subsection{Sources with and without a disk-like dust distribution}

Soon after the discovery of the crystalline silicates, it became clear that all sources, which show a high abundance of crystalline silicates, have a disk-like circumstellar dust distribution (Molster et al. 1999a). It should be noted that the presence of a disk does not guarantee a high abundance of crystalline silicates (Fig. 3). This relation only works one way. The reason behind the higher fraction of crystalline silicates in disk sources is still under investigation. The thermal annealing timescales for dust at $100 \mathrm{~K}$ (the typical dust temperature in these disks) is many orders of magnitude longer than the age of the disks (Hallenbeck, Nuth \& Daukantas 1998). Several mechanisms for the temperature crystallization have been proposed, like local heating by lightning, impacts of high energetic particles (Molster et al. 1999a) or internal heating by radioactive decay of ${ }^{26} \mathrm{Al}$ (Speck et al. 2002). 


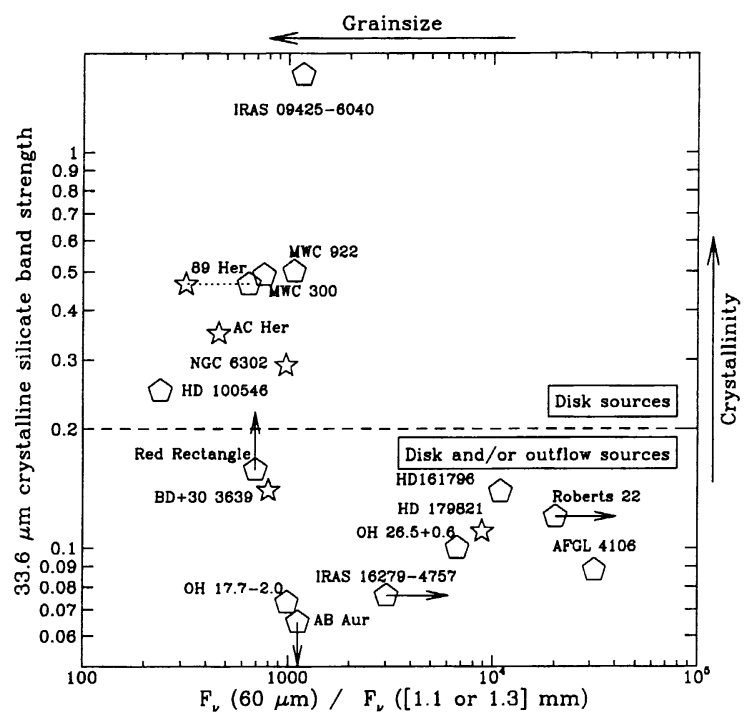

Figure 3. The ratio of the IRAS $60 \mu \mathrm{m}$ flux to the mm continuum flux versus the relative strength of the $33.6 \mu \mathrm{m}$ olivine feature. The stars denote measurements at $1.1 \mathrm{~mm}$, while the pentagons denote measurements at $1.3 \mathrm{~mm}$. Figure taken from Molster et al. (1999a).

Apart from the strength differences of the spectral features, there are also spectral shape differences between evolved stars with and without a disk-like dust distribution (see Molster et al. 2002a).

\subsection{Model fits and correlations}

Molster et al. (2002b) fitted the continuum subtracted dust spectra of 12 evolved stars, with a two component (forsterite and enstatite) dust model. An example of these fits is given in Fig. 4. It shows that these 2 components already fit most of the features in the spectrum very well. From these fits a typical temperature for forsterite and enstatite has been derived. The temperatures derived for the enstatite and forsterite grains are similar (see Fig. 18 of Molster et al. 2002b). This could indicate that both type of grains are in thermal contact and formed together. However, the near-IR optical constants of enstatite are not well known and it is also possible that the absorptivity/emissivity of forsterite and enstatite are rather similar, in which case both type of grains would be at the same temperature even without thermal contact. New absorption measurements of enstatite should solve this problem.

Based on these simple model fits, one can also derive a mass ratio of these two crystals (see Fig. 20 of Molster et al. 2002b). Based on 9 disk sources and 3 outflow (or non-disk) sources Molster et al. (2002b) found that enstatite is on average 3 times as abundant as forsterite. There is some evidence that in the outflow sources this mass ratio is even higher, although it should be noted that this is only based on 3 sources and more observations are required. 


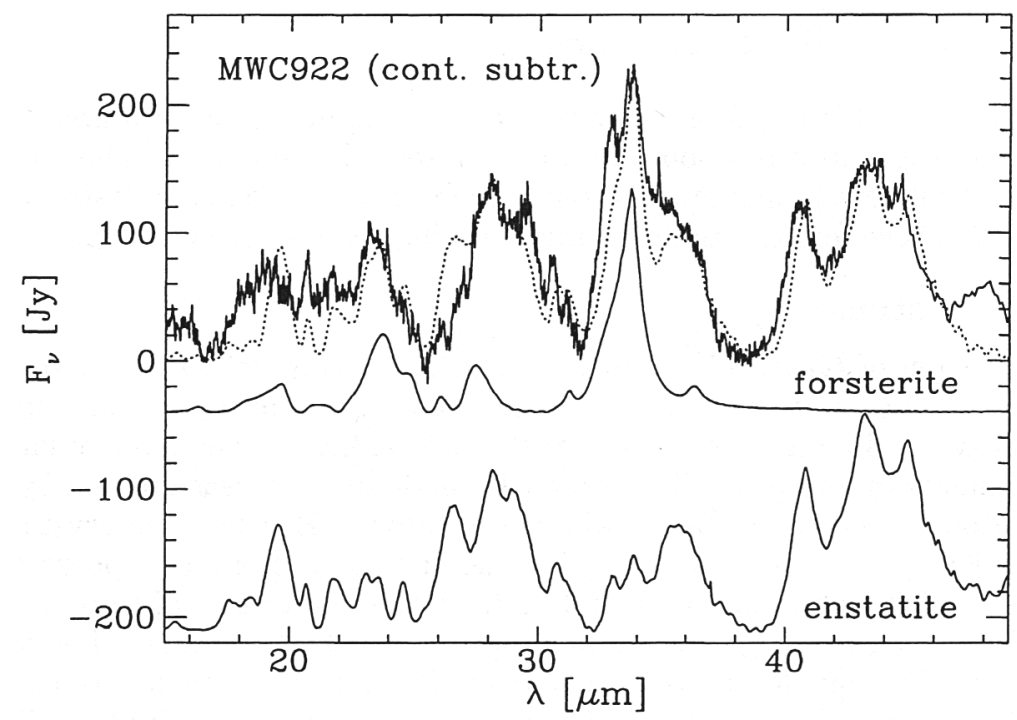

Figure 4. The model fit (dotted line) of the continuum subtracted spectrum of MWC 922 (solid line). The model fit is a combination of forsterite of $90 \mathrm{~K}$ and enstatite of $100 \mathrm{~K}$ (Molster et al. 2002b).

The typical temperature of amorphous silicates, derived from fits to the continuum, is higher than that of the forsterite grains (see Fig. 19 of Molster et al. 2002b). This indicates that the two grain populations are either not in thermal contact or they have a different spatial distribution. 1D Radiative transfer calculations show that the temperature difference is consistent with a cospatial distribution of separate grain populations (e.g. Molster et al. 1999b,2001). However, there is also evidence that the amorphous and crystalline silicates do not have the same spatial distribution. Sylvester et al. (1999) find that for the $\mathrm{OH} / \mathrm{IR}$ star OH104.9+2.4 the crystalline silicate features are already in emission while the amorphous silicates are still in absorption. This measurement excludes the possibility that the crystalline silicates are colder simply, because they are at larger distances. The most likely explanation is that the crystalline silicates are mainly located in the denser equatorial region, while the amorphous silicates have a more spherical distribution and unless looking edge on will cause the foreground absorption. Unfortunetaly, it is not yet possible to image the spatial distribution of the crystalline silicates. This is an area for future research. The answer to the spatial distribution of the crystalline silicates will help us to understand the dust formation much better.

Crystalline silicates are not only very helpful in the investigation of the formation of dust and their dependence on the local conditions (like temperature (gradient), chemistry and density), they can also directly be used as tracers of the present dust conditions. Bowey et al. (2002) show that the position and FWHM of the forsterite feature at $69.0 \mu \mathrm{m}$ can be used as a temperature indicator of the circumstellar dust shell. 


\section{Crystalline silicate evolution}

Crystalline silicates are found around both young and evolved stars. However, there is an upperlimit of about $2 \%$ for the interstellar medium. Therefore, most of the crystalline silicates found around evolved stars are likely destroyed in the ISM, while those found around young stars have to be formed in situ.

\subsection{Formation}

The formation of (crystalline) silicates is still not completely understood. But, also here the infrared spectra seem to reveal part of the secret. The spectra in Fig. 5 show the 33 micron complex for three different objects and two laboratory measurements of forsterite. The forsterite made in Jena, was created by slowely cooling down a melt so that it would crystallize. Because the crystallization started likely at different places inside the crystal, it became a polycrystalline material, which whas some crystal defects at the boundaries of these individual crystal elements, making the 33.6 micron feature a little broader. The forsterite from Japan is a single crystal and shows therefore a much narrower feature. Vapor phase growth processes will lead to single crystals (Bradley, Brownlee \& Veblen 1983). This last process is expected to take place in the outflows of evolved stars, where dust will condense out of the gas-phase at temperatures above the annealing temperature ( $\pm 900 \mathrm{~K}$; Hallenbeck et al. 1998). The spectra of evolved stars show indeed a rather narrow 33.6 micron features. HD100546 is a young star, which shows a relatively broad 33.6 micron feature. Here, most crystalline silicates are expected to be formed via a melt of an amorphous silicate close to the central star (Bouwman et al. 2001). The shape of the 33 micron complex in this young star is similar to the Jena forsterite and therefore in agreement with its expected formation history. More research is still necessary in this area, but the shape of the features seem to contain information about the formation processes.

Another question about the formation of silicates around evolved stars still stands: "How is the amorphous dust formed?" The high temperature at which the first silicates form, is high enough to anneal them. So, either there is a lot of dust formed below the annealing temperature, or the crystalline silicates are made amorphous afterwards, e.g. by the implantation of Fe (Tielens et al. 1998). An accurate determination of the spatial distribution of the amorphous and crystalline silicates will again help to solve this problem.

\subsection{Destruction}

Crystalline silicates are not found in the ISM. Therefore the crystalline silicates formed around evolved stars are likely destroyed in the ISM. Demyk et al. (2001) showed that supernova shock waves are very capable in destroying most of the crystalline silicates.

\section{Conclusions}

Thanks to the high spectral resolution of ISO, it has now become possible to do real astromineralogy. Mineralogical compositions can now accurately be determined, and also other information, like temperature and formation history can 


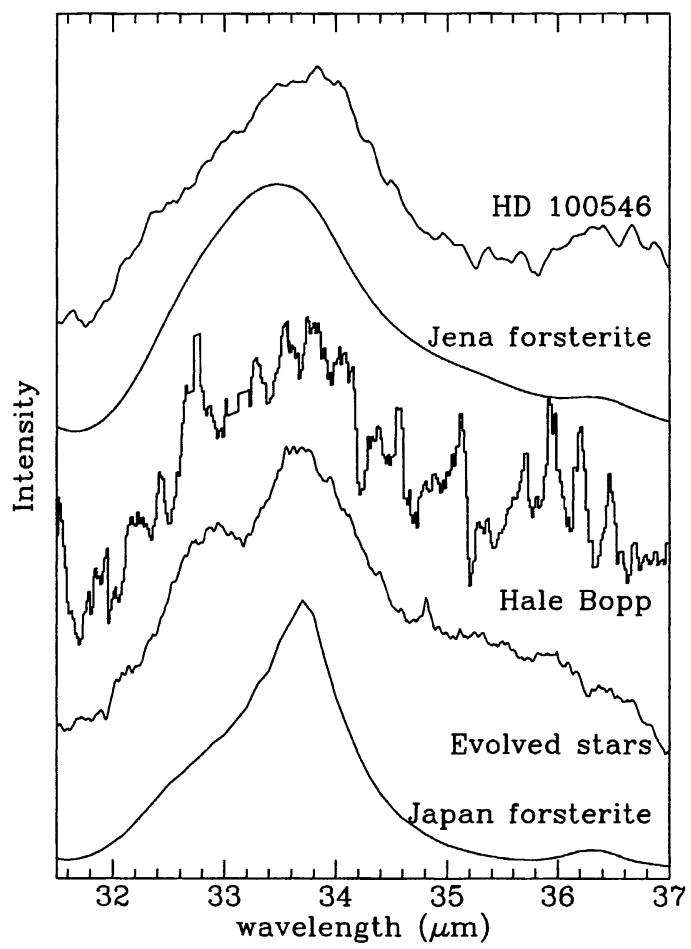

Figure 5. The 33 micron complex of the young star HD100546 (Malfait et al. 1998), the comet Hale Bopp (Crovisier et al. 1997) and an average of the 33 micron complexes of evolved stars with a disk (Molster et al. 2002a), together with 2 laboratory measurements of forsterite one from Jena (Jäger et al. 1998) and one from Japan (Koike et al. 2000b). (Figure taken from Molster et al. 2002b).

be retrieved from the spectral shape of the dust spectrum. Crystalline silicates around evolved stars are (almost) Fe-free, i.e. forsterite and enstatite. Besides the retrieval of their exact mineralogical composition, which already gives important clues about the dust formation process, there is also evidence that the shape of the features contains information about the formation history. To fully understand the spectra, new research in the laboratory as well as new infrared, spectral and spatial, observations are necessary. The coming launch of SIRTF will definately help to extend the database of spectra and solve some of the pending questions.

\section{References}

Bouwman, J., Meeus, G., de Koter, A., et al. 2001, A\&A375, 950

Bowey, J.E., Lee, C., Tucker, C., et al., 2000, in ISO beyond the peaks: The 2nd ISO workshop on analytical spectroscopy. Eds. A. Salama, M.F.Kessler, K. Leech \& B. Schulz. ESA-SP 456, 339 
Bowey, J.E., Barlow, M.J., Molster, F.J., et al. 2002, MNRAS in press ISO beyond the peak, ed A. Salama, ESA SP-456, 339

Bradley, J.P., Brownlee, D.E. \& Veblen, D.R. 1983, Science 301, 473

Crovisier, J., Leech, K., Bockelee-Morvan, D., et al. 1997, Science 275, 1904

Demyk, K., Carrez, P., Leroux, H., et al. 2001, A\&A368, L38

Dorschner, J., Begemann, B., Henning, T., et al. 1995, A\&A300, 503

Hallenbeck, S.L., Nuth, J.A., \& Daukantas, P.L. 1998, Icarus 131, 198

Jäger, C., Molster, F.J., Dorschner, J., et al. 1998, A\&A339, 904

Kemper, F., Waters, L.B.F.M., de Koter, A. \& Tielens, A.G.G.M. 2001, A\&A369, 132

Kemper, F., Jäger, C., Waters, L.B.F.M., et al. 2002a, Nature 415, 295

Kemper, F., de Koter, A., Waters, L.B.F.M., et al. 2002b, A\&A in press

Kessler, M.F., Steinz, J.A., Anderegg, M.E., et al. 1996, A\&A315, L27

Koike, C., Tsuchiyama, A., Shibai, H., et al. 2000a, A\&A363, 1115

Koike, C., Chihara, H., Tsuchiyama, A., et al. 2000b, in Proceedings of the 33rd ISAS Lunar and Planetary Symposium, p 95

Malfait, K, Waelkens, C., Waters, L.B.F.M., 1998, A\&A332, L25

Mennella, V., Brucato, J.R., Colangeli, L., et al. 1998, ApJ 496, 1058

Molster, F.J., Yamamura, I., Waters L.B.F.M., et al. 1999a, Nature 401, 563

Molster, F.J., Waters, L.B.F.M., Trams, N. et al. 1999b, A\&A350, 163

Molster, F.J., Yamamura, I., Waters, L.B.F.M., et al. 2001, A\&A366, 923

Molster, F.J., Waters, L.B.F.M., Tielens, A.G.G.M. 2002a, A\&A in press

Molster, F.J., Waters, L.B.F.M., Tielens, A.G.G.M., et al. 2002b, A\&A in press

Speck, A.K., Kemper, F., Whittington, A.G., et al. 2002, Lunar Planet. Sci. XXXIII, abstract nr: 1197

Sylvester, R.J., Kemper, F., Barlow, M.J. et al., 1999, A\&A352, 587

Tielens, A.G.G.M., Waters, L.B.F.M., Molster, F.J. and Justtanont, K. 1998, ApSS 255,415

Waters, L.B.F.M., Molster, F.J., de Jong, T., et al. 1996, A\&A315, L361

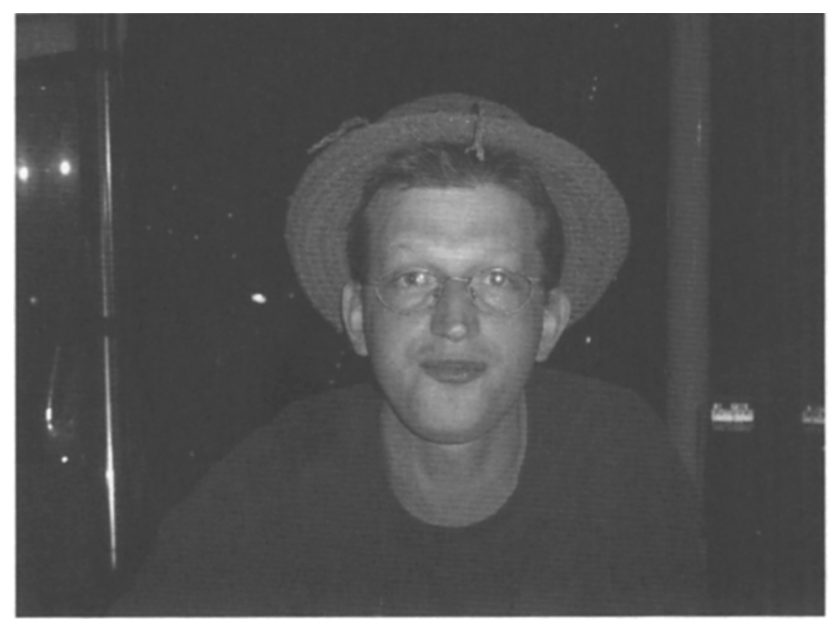

Figure 6. F.J. Molster (photo courtesy of O. de Marco). 